\title{
Surface-plasmon-enhanced light scattering from microscopic spheres
}

\author{
M. J. Jory, ${ }^{\text {a) }}$ P. S. Cann, and J. R. Sambles \\ Thin Film Photonics Group, School of Physics, University of Exeter, Stocker Road, Exeter, \\ EX4 4QL United Kingdom \\ E. A. Perkins \\ Detection, Dstl, Porton Down, Salisbury, Wilts., SP4 OJQ United Kingdom
}

(Received 20 March 2003; accepted 24 July 2003)

The enhanced light scattering from microscopic latex spheres placed in the optical field associated with a surface-plasmon resonance is explored. Spheres of $200 \mathrm{~nm}$ diameter are placed on an optically thin gold film that supports the surface-plasmon and the scattered intensity is then measured as a function of scattering angle. This is compared to the scattering profiles obtained from spheres placed on a bare glass substrate. In both cases, the experimental data are compared to theory. This system is of interest in the field of optical biosensing. (C) 2003 American Institute of Physics.

[DOI: $10.1063 / 1.1613798$ ]

Light striking a planar glass/air interface at an angle of incidence greater than the critical angle undergoes total internal reflection and consequently there is no transmitted ray. However, placing a scattering particle in the evanescent field that decays into the air half-space (i.e., close to the interface) allows light to radiate in an otherwise totally dark environment. The evanescent field generated by a $p$-polarized beam at a glass/air interface has a maximum amplitude $\sim 3$ times the incident field. One variant of this simple idea includes an optically thin metal layer coating on the glass surface that allows the excitation of a surface-plasmon polariton resonance (SPR). ${ }^{1}$ The amplitude (evaluated just above the metal surface) of the optical fields associated with the SPR, that decay exponentially into the surrounding media, are typically (e.g., when using a gold film illuminated by $632.8 \mathrm{~nm}$ wavelength radiation) $\sim 10$ times the amplitude of the incident field. ${ }^{1}$ Therefore, it may be possible to visualize a single particle in the SPR field more easily than one on a bare glass substrate.

Here, we compare the scattering response of latex microspheres placed behind a glass/air interface to those placed behind an optically thin gold film that supports the SPR. A 5 $\mu l$ drop of a microsphere/isopropyl alcohol (IPA) suspension is placed onto a (Corning 7509) fusion-drawn glass slide (surface roughness $\sim 13 \mathrm{~nm}$ ). The slide has previously been refractive index matched to a prism so that, once the IPA has evaporated, the spheres may be illuminated through the glass using the $10 \mathrm{~mW} p$-polarized beam from a HeNe laser incident at an angle of $45^{\circ}$ (beyond critical angle). (The laser beam spot is $\sim 100 \mu \mathrm{m}$ in diameter at the sphere-covered surface.) Scattered intensity versus scattering angle is now recorded. Next, a $5 \mu \mathrm{l}$ drop of a second microsphere/IPA suspension, higher in concentration than the first, is applied to the sample surface. Light scattering data are again acquired. This process is repeated using suspensions successively higher in concentration. (The spheres are deposited on the glass slide in situ in order to avoid disturbing the optical arrangement between measurements.)

${ }^{a)}$ Electronic mail: mjjory@exeter.ac.uk
Figure 1(a) compares the shapes of scattering profiles obtained having deposited $200 \mathrm{~nm}$ diameter latex spheres from suspensions of concentrations $6.3 \times 10^{-5}, 8.4 \times 10^{-5}$, $1.05 \times 10^{-4}$, and $2.1 \times 10^{-4} \mathrm{~g} / \mathrm{cm}^{3}$ in succession. (The response of the bare substrate is first subtracted from the data acquired for each sphere-covered surface. Arbitrary scaling and an adjacent-point average are then performed to obtain the data shown here.) All five curves are very similar in shape with a large peak in scattered intensity at $60^{\circ}$ and a smaller peak at $-65^{\circ}$. The consistent shape of the scattering profiles implies that the spheres are behaving as independent scatterers, provided the suspensions used are of concentration $\leqslant 2.1 \times 10^{-4} \mathrm{~g} / \mathrm{cm}^{3}$. It should be noted that the fine structure in the data is not random noise. For an individual deposition of spheres consecutive angle scans reveal that the

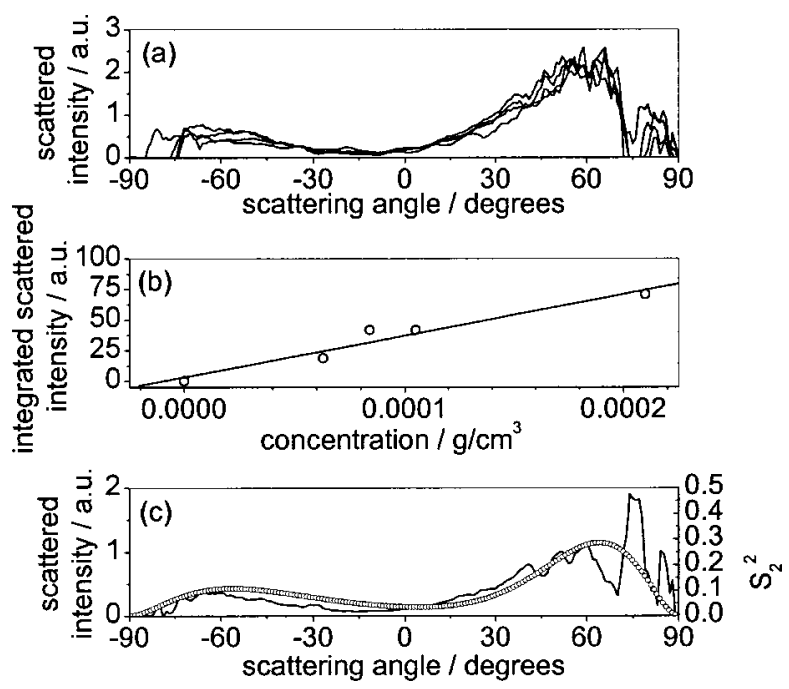

FIG. 1. (a) Comparison of angle-dependence of scattered intensity (arbitrary scaling factors) for $200 \mathrm{~nm}$ diameter latex spheres behind a glass/air interface and illuminated with $p$-polarized light incident at $45.0^{\circ}$ (beyond the critical angle). Each curve represents a different concentration of the sphere/ IPA suspension used to deposit the spheres. (b) Scattered intensity, integrated as a function of scattering angle vs concentration of sphere/IPA suspension (circles). Line: linear fit. (c) Scattered intensity vs scattering angle. Line: experiment (using a suspension of concentration $2.1 \times 10^{-4} \mathrm{~g} / \mathrm{cm}^{3}$ ). Circles: response predicted by theory ( $S_{2}^{2}$ is proportional to intensity). 
pattern is highly repeatable. In this work, the laser beam spot strikes many spheres at once. Consequently, as scattered light is simultaneously collected from more than one sphere this structure may be due to interference effects, i.e., the spheres form a planar distribution of coherent sources. This suggestion is supported by the fact that the deposition of additional spheres on the surface not only increases the scattered intensity but also changes the pattern of the fine structure. Figure 1(b) shows scattered intensity, integrated as a function of scattering angle versus concentration (circles). If the spheres are behaving as independent scatterers a linear relationship should be observed (the line is a linear fit). To first order there is clearly a simple relationship although there are some discrepancies between the data and the linear fit.

To first approximation, the amplitude of the decaying evanescent field may be considered constant over the entire sphere provided its decay length $\left(\approx \lambda_{0}\right.$, where $\lambda_{0}$ is the wavelength in free-space of an optical beam incident just beyond the critical angle) $\gg$ sphere radius. In Fig. 1(c) the experimentally measured scattering response (solid line) of the system (having deposited spheres onto a bare substrate from a $2.1 \times 10^{-4} \mathrm{~g} / \mathrm{cm}^{3}$ suspension) is compared to the response predicted by established theory (circles) describing light scattering from a single sphere behind a planar interface $^{2}\left(S_{2}^{2}\right.$ is proportional to intensity). The effect of varying the values for sphere refractive index and sphere diameter in the theory is explored. Use of the given values of $1.60 \pm 0.05$ and $(200 \pm 5) \mathrm{nm}$ for refractive index and diameter, respectively, provide the best agreement between theory and experiment. To obtain a detailed comparison between the two profiles all data are plotted on a linear scale. There is good agreement between the shapes of the two curves and the positions of the intensity peaks. However, the minimum in scattered intensity that is theoretically predicted to occur at $4.9^{\circ}$ occurs at $-9^{\circ}$ in the experimental data.

The fusion-drawn glass slide is now replaced with a glass slide that has been coated in a gold film ${ }^{3}$ of thickness $\sim 45 \mathrm{~nm}$ (surface roughness $\sim 5 \mathrm{~nm}$ ). An angle-of-incidence ( $43.5^{\circ}$ in the glass) is selected that allows excitation of SPR at the gold/air interface. Figure 2(a) shows the scattering profiles obtained after deposition of $200 \mathrm{~nm}$ diameter latex spheres on the gold surface. Suspensions of concentration $2.1 \times 10^{-5}$ (bottom curve), $4.2 \times 10^{-5}, 6.3 \times 10^{-5}, 8.4$ $\times 10^{-5}, 1.05 \times 10^{-4}$, and $2.1 \times 10^{-4} \mathrm{~g} / \mathrm{cm}^{3}$ (top curve) are used. (The signal obtained from the bare gold surface is subtracted from the scattering response of the sphere-covered surfaces.) A comparison of the shapes of the curves is shown in Fig. 2(b). It is clear that the spheres are behaving as independent scatterers for suspensions of concentration $\leqslant 2.1$ $\times 10^{-4} \mathrm{~g} / \mathrm{cm}^{3}$. A near-linear relationship between integrated scattered intensity and concentration is shown in Fig. 2(c).

Figure 3 shows scattered intensity (integrated as a function of scattering angle) versus angle of incidence measured using a bare gold surface (circles), i.e., no spheres present. The line shows the amplitude, as predicted by theory, ${ }^{4}$ of the evanescent field (arbitrary scaling factor) generated at the metal/air interface for a 45-nm-thick gold film of optical permittivity $-11.5+1.51 i$. SPR excitation is observed as a clear peak in intensity at $43.5^{\circ}$ in both curves and there is good agreement between the peak widths at half maximum. Downloaded 27 May 2008 to 144.173.6.22. Redistribution subject
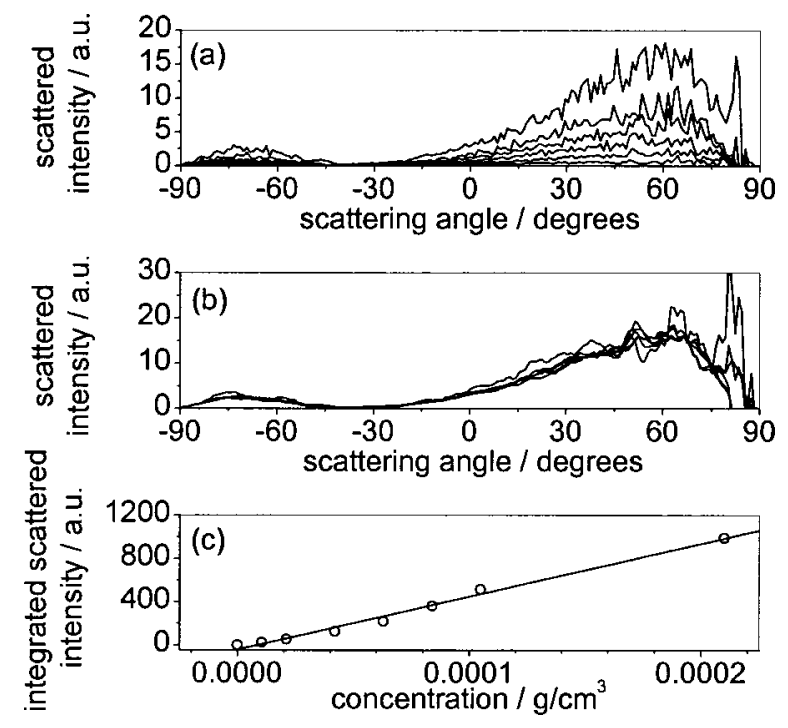

FIG. 2. (a) Total scattered intensity vs scattering angle for $200 \mathrm{~nm}$ diameter latex spheres placed in the optical fields associated with SPR excited on a gold film. The spheres are deposited using sphere/IPA suspensions of concentration $2.1 \times 10^{-5}$ (bottom curve), $4.2 \times 10^{-5}, 6.3 \times 10^{-5}, 8.4 \times 10^{-5}$, $1.05 \times 10^{-4}$, and $2.1 \times 10^{-4} \mathrm{~g} / \mathrm{cm}^{3}$ (top curve). (b) Comparison of the angledependence of scattered intensity (arbitrary scaling factors). (c) Scattered intensity, integrated as a function of scattering angle vs concentration of sphere/IPA suspension. Circles: experiment. Line: linear fit.

We now extend the theory ${ }^{2}$ to include the transmissivity and reflectivity coefficients for a stratified isotropic planar structure. ${ }^{4}$ This allows the optically thin gold film to be included in the model and the scattered fields that are reflected at the air/gold interface to be accounted for. Figure 4 shows the measured scattering profile for a sphere-covered gold surface (solid line) (using a $2.1 \times 10^{-4} \mathrm{~g} / \mathrm{cm}^{3}$ suspension). The main peak in scattered intensity, that occurs at $69^{\circ}$, has a height of 17.5 [much higher than that obtained from spheres placed on bare glass (1.9), Fig. 1(c)]. These data are now compared to the theoretically predicted response using the extended theory (open circles). Sphere refractive index (1.6 $\pm 0.05)$ and diameter $[(200 \pm 5) \mathrm{nm}]$, angle-of-incidence $\left[(43.5 \pm 0.1)^{\circ}\right]$ along with the gold film thickness $[(45$ $\pm 1) \mathrm{nm}]$ and optical permittivity $[-11.5( \pm 0.5)+(1.5$ $\pm 0.1) i]$ are adjusted in the theory to provide the best fit to data (the best fit values are given in brackets). The height of the main peak in scattered intensity that occurs at $69^{\circ}$ is 10.2 , again much higher than the value (0.3) predicted by theory for spheres on a bare glass substrate in Fig. 1(c). There is good agreement between the positions of the peaks in scattered intensity recorded in the experimental data and those predicted by theory. A minimum in scattered intensity

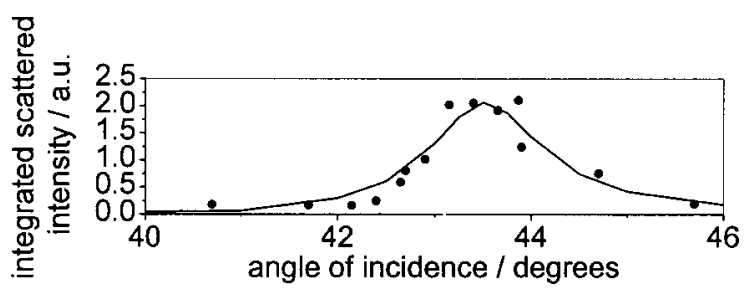

FIG. 3. Scattered intensity, integrated as a function of scattering angle vs angle-of-incidence for a $p$-polarized input beam striking a bare gold film (circles). The solid line represents the evanescent field intensity as predicted by theory (arbitrary scaling factor). 


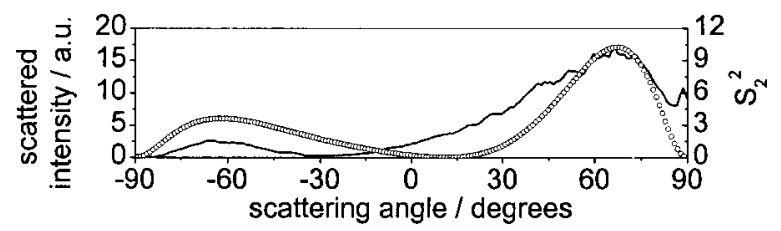

FIG. 4. Scattered intensity vs scattering angle. Line: experiment (sphere/IPA suspension of concentration $\left.2.1 \times 10^{-4} \mathrm{~g} / \mathrm{cm}^{3}\right)$. Circles: extended theory $\left(S_{2}^{2}\right.$ is proportional to intensity).

is also observed in both curves although there is a large discrepancy between the experimentally measured position $\left(-32^{\circ}\right)$ and that predicted by theory $\left(11^{\circ}\right)$. This may be due to fields that are first scattered from the sphere and reflected from the gold film to then scatter from the sphere for a second time not being included in the model. ${ }^{2}$ (Reflectivities at near-normal angles-of-incidence for the gold film will be $\sim 80 \%$ compared to $\sim 4 \%$ for the air/glass interface studied previously.)

In summary, the scattering response of $200 \mathrm{~nm}$ diameter latex spheres placed in $p$-polarized evanescent fields has been studied. Although many scatterers were optically sampled at once, the measured intensity profiles indicate "independent scattering" behavior. Data acquired from spheres on a bare glass substrate were compared with established theory. Good agreement between the angular positions, and relative heights of the intensity peaks was observed. However, a difference of $14^{\circ}$ was recorded in the position of the intensity minimum measured experimentally and that predicted by theory. Angle-dependent scattering profiles were also recorded for latex spheres placed in an SPR field and compared to the response predicted by extended theory. Again, good agreement between the positions of the intensity peaks was observed, but the discrepancy in intensity minimum position, increased to $43^{\circ}$.

Better agreement between experiment and theory may be obtained by including the effects of secondary scattering in the theory. Alternatively, using infrared radiation would decrease the effects of surface roughness of the substrate. This would also allow a sphere, or spheres of larger radius but smaller size parameter (i.e., sphere circumference divided by optical wavelength) to be used.

Conventional optical biosensors ${ }^{3}$ incorporate detection systems that collect light radiated within a cone of solidangle centered on a normal to the sensor surface. Here, however, both experiment and theory indicate that the scattered intensity is maximal at $\sim 60^{\circ}$ scattering angle. It may therefore be possible to obtain better contrast by adapting the sensor collection optics to take account of this.

Exciting SPR along the interface occupied by the latex spheres increased the integrated scattered intensity by a factor of $\sim 14$, as measured by experiment, approximately half the increase (a factor of $\sim 29$ ) predicted by a simple theory that ignores second-order scattering from the sphere. However, it is clear that excitation of SPR on a gold film allows the intensity of scattered light to be increased by at least an order of magnitude. As the latex spheres used in this work are similar to biological particles in both size and refractive index this may have important ramifications in the field of optical biosensing. ${ }^{3}$

The authors gratefully acknowledge Dstl and EPSRC for funding.

\footnotetext{
${ }^{1}$ H Raether, Surface Plasmons (Springer, Berlin, 1988).

${ }^{2}$ G. Videen, J. Opt. Soc. Am. A 10, 110 (1993).

${ }^{3}$ E. A. Perkins and D. J. Squirrell, Biosens. Bioelectron. 14, 853 (2000).

${ }^{4}$ R. M. A. Azzam and N. M. Bashara, Ellipsometry and Polarized Light (Elsevier, The Netherlands, 1996).
} 